\title{
COMPORTAMIENTO DEL CONSUMIDOR EN LA CO-CREACIÓN DE VALOR Y SU RELACIÓN CON LA SATISFACCIÓN EN EL ENTORNO UNIVERSITARIO: UNA APLICACIÓN A LA UNIVERSIDAD DE IBAGUÉ (COLOMBIA)*
}

\author{
BLANCA ASTRID MORENO DE CASTRO"* \& MARÍA HAYDÉE CALDERÓN GARCÍA"* \\ UNIVERSIDAD DE IBAGUÉ (COLOMBIA)-UNIVERSIDAD DE VALENCIA (ESPAÑA)
}

Recibido/ Received/ Recebido: 08/10/2015-Aceptado/ Accepted / Aprovado: 04/07/2016

\section{Resumen}

Aportes académicos señalan que el consumidor es el actor más importante en la co-creación de valor, pero pocos estudios se han centrado en descubrir y comprobar la influencia de los comportamientos del consumidor que co-crea valor en su satisfacción. En consecuencia, en este artículo se contrasta esta relación en el contexto universitario, para lo cual se plantea un modelo relacional con las variables: comportamiento del consumidor en la co-creación de valor y satisfacción. Dicho modelo se aplicó a una muestra representativa de estudiantes de una universidad colombiana. Los resultados evidencian algunas relaciones positivas. La contribución teórica del estudio radica en que esta es la primera vez que se analiza la influencia de la co-creación desde la perspectiva del consumidor en la satisfacción en el contexto universitario y en un país latinoamericano.

Palabras clave: Co-creación de valor; Interacciones; Satisfacción; Estudiantes; Universidad.

\section{CONSUMER BEHAVIOR IN THE CO-CREATION OF VALUE AND ITS RELATIONSHIP WITH SATISFACTION IN THE UNIVERSITY ENVIRONMENT: AN APPLICATION TO THE UNIVERSITY OF IBAGUÉ (COLOMBIA)}

\begin{abstract}
Academic contributions indicate that the consumer is the most important actor in the co-creation of value. But few studies have focused on discovering and check the influence of consumer behaviors that co-creates value in her/his satisfaction. Accordingly, this article contrasts this relationship in the university context for which a relational model with variables arises: consumer behavior in co-creating value and satisfaction. This model was applied to a representative sample of students from a Colombian university. The results show some positive relationships. The theoretical
\end{abstract}

Artículo de investigación derivado del Doctorado en Marketing de la Universidad de Valencia (España).

Docente de la Universidad de Ibagué. Doctoranda en Marketing de la Universidad de Valencia. Dirección postal: Carrera 22, Calle 67 Avenida Ambalá. Facultad de Ciencias Económicas y Administrativas, Universidad de Ibagué, Ibagué (Colombia). Teléfono: +57(8)2709400, fax: +57(8)2709443. Correo electrónico: blanca.moreno@unibague.edu.co

*** Docente de la Universidad de Valencia. Correo electrónico: haydee.calderon@uv.es 
contribution of the study is that this is the first time the influence of co-creation is analyzed from the perspective of consumer satisfaction in the university context and in a Latin American country. Keywords: Co-creation of value; interactions; Satisfaction; Students; College.

\title{
COMPORTAMENTO DO CONSUMIDOR NA CO-CRIAÇÃO DE VALOR E SUA RELAÇÃO COM A SATISFAÇÃO NO MEIO UNIVERSITÁRIO: UMA APLICAÇÃO À UNIVERSIDADE DE IBAGUÉ (COLÔMBIA)
}

\begin{abstract}
Resumo
Estudos acadêmicos indicam que o consumidor é o agente mais importante na co-criação de valor, mas poucos estudos se centraram em descobrir e comprovar a influência dos comportamentos do consumidor que co-cria valor em sua satisfação. Neste artigo contrasta-se esta relação no contexto universitário, para o qual propõe-se um modelo relacional com as variáveis: comportamento do consumidor na co-criação de valor e satisfação. Este modelo foi aplicado em uma mostra representativa de estudantes de uma universidade colombiana. Os resultados evidenciam algumas relações positivas. A contribuição teórica do estudo revela que esta é a primeira vez que se analisa a influência da co-criação desde a perspectiva do consumidor na satisfação no contexto universitário e em um país latino-americano.
\end{abstract}

Palavras chave: Co-criação de valor; Interações; Satisfação; Estudantes; Universidade.

Moreno, B. \& Calderón, H. (2017). Comportamiento del consumidor en la co-creación de valor y su relación con la satisfacción en el entorno universitario: una aplicación a la universidad de Ibagué (Colombia). En: Revista de la Facultad de Ciencias Económica: Investigación y Reflexión. rev.fac.cienc.econ, XXV (1). DOI: http://dx.doi.org/10.18359/rfce.1193

JEL: $M 31, M 39$.

\section{Introducción}

El presente artículo está centrado en estudiar la influencia que tienen algunos comportamientos del estudiante universitario en la satisfacción que dicho estudiante percibe con su universidad, teniendo como referentes teóricos los estudios de Yi \& Gong (2013) sobre comportamientos de los consumidores que posibilitan la co-creación de valor con las empresas, así como estudios relacionados con la satisfacción del estudiante tales como los de Helgesen \& Nesset (2007) y González et al. (2016), entre otros.

Para Prahalad \& Ramaswamy (2004) y, posteriormente, para Payne, Storbacka \& Frow (2008), la creación de valor está experimentando un cambio de paradigma pasando de ser entendida desde el producto y dentro de la firma, a ser entendida como el resultado de la interacción entre la empresa y el consumidor, de manera que es este último quien crea valor a través de sus experiencias personalizadas con los productos o servicios de las empresas. A ellos se suman Grönroos \& Voima (2013) al afirmar que los consumidores participan en el marketing, el consumo y la creación del proceso de valor y que el consumidor en vez de co-crear valor es quien lo crea, con o sin interacciones con las empresas.

Por su parte, tanto el constructo satisfacción como sus causas y consecuencias han sido estudiados am- 
pliamente en la literatura del marketing, dada su importancia para la sostenibilidad y permanencia de las empresas. Sin embargo, para el contexto de esta investigación, destacamos los trabajos realizados sobre el constructo satisfacción del estudiante (Elliott \& Shin, 2002), la imagen de marca de la institución universitaria y su relación con la satisfacción estudiantil (Palacio, Meneses \& Pérez, 2002; Schlesinger, Cervera \& Pérez, 2016), modelos de satisfacción estudiantil que contemplan aspectos de las comunicaciones en el marketing (Jurkowitsch, Vignali \& Kaufmann, 2006), los conductores de la actividad estudiantil para alcanzar la satisfacción (Helgesen \& Nesset, 2007), los satisfactores de los estudiantes en la educación superior (Butt, 2010), los factores claves que determinan la satisfacción estudiantil en la educación a distancia (Ali \& Ahmmad, 2011), los criterios para la evaluación de la satisfacción de los estudiantes (Zineldin, Akdag \& Vasicheva, 2011) y las percepciones de la calidad de servicio en las instituciones de educación superior (Cardona \& Bravo, 2012), entre otras, pues se constituyen en insumos valiosos para nuestra reflexión al respecto.

Recientemente, en los estudios realizados por Vega, Revilla \& Cossío (2013) y Yi \& Gong (2013) se resalta la necesidad de fortalecer la teoría del marketing a través del análisis de la relación entre el comportamiento del consumidor en la co-creación de valor, y la satisfacción del consumidor en diversos contextos y áreas geográficas. Acogiendo lo expresado por estos autores, decidimos centrar nuestra investigación en el ámbito universitario, pues analizar la satisfacción del estudiante universitario es importante dada la influencia que tiene la satisfacción en la lealtad y la permanencia en la universidad, así como en el papel de prescriptores que pueden asumir los estudiantes ante futuros universitarios.

Es así como el aporte académico del estudio radica en fortalecer el estudio de la co-creación de valor desde la perspectiva del consumidor, testando una escala de medición creada recientemente, para determinar, por primera vez, la posible influencia que dichos comportamientos de co-creación puedan tener en la satisfacción del estudiante de educación superior, en el contexto colombiano.
Con base en lo expuesto, con la certeza de que existe la necesidad de un fortalecimiento de la base académica que muestre la relación entre la participación del consumidor en la co-creación de valor y la satisfacción percibida en el ámbito universitario, el presente artículo tiene por objetivos analizar la participación del estudiante universitario en la cocreación de valor en una universidad, y analizar la relación de dicho comportamiento de co-creación con la satisfacción que éste percibe con el servicio ofrecido por su institución de educación superior.

Para alcanzar los objetivos propuestos, el artículo está compuesto por cuatro apartados: marco conceptual, metodología, resultados, y, conclusiones e implicaciones. En el marco conceptual se hace una revisión teórica de los constructos co-creación de valor y satisfacción y se introducen las hipótesis. En el segundo apartado se describe la metodología del estudio empírico cuantitativo, el instrumento a utilizar, el proceso de recolección de la información, las escalas y el modelo relacional del estudio. Posteriormente, se define el método estadístico para el análisis de la información recolectada y se presentan los resultados. Por último se presentan las conclusiones, implicaciones del estudio y sugerencias para futuras investigaciones.

\section{Marco conceptual y planteamiento de hipótesis}

La co-creación de valor es un constructo relativamente nuevo y ha sido enunciado de diversas maneras en la teoría del marketing, aunque la actividad en sí misma haya sido practicada desde hace largo tiempo (Ind \& Coates, 2013; Grönroos, Strandvik \& Heinonen., 2015). Las nuevas formas de interacción entre consumidores y empresas exigen de los directivos entender los ambientes y ecosistemas del consumidor para generar actividades de co-creación de valor en que los consumidores tengan espacio para participar activamente (Saarijärvi, Kannan \& Kuusela, 2013); en consecuencia, las empresas están comprometidas a desarrollar competencias que permitan la integración de recursos, con sus consumidores, otros actores y con la sociedad en general, para promover acciones de co-creación de valor que faciliten el cumplimiento de las promesas de valor 
(Bettencourt et al., 2014). Es así como la co-creación de valor surge a través del diálogo y la transferencia de conocimiento y de otros recursos e interacciones que promueven el aprendizaje organizacional y la creación y renovación de recursos. La integración de recursos se presenta cuando los actores unen sus recursos para beneficio mutuo de acuerdo a sus expectativas, necesidades y capacidades (Gummesson \& Mele, 2010), especialmente de habilidades y conocimientos (Ballantyne \& Varey, 2008).

Prahalad \& Ramaswamy (2004) definen la co-creación de valor como la creación conjunta de valor entre la firma y el consumidor, a través de interacciones de calidad que permiten al consumidor individual crear experiencias únicas con la empresa. Más adelante Vargo \& Lusch (2011), Neghina et al. (2015), y Grönroos (2011), amplían el concepto aduciendo que la co-creación de valor, que puede ser para una o para ambas partes, se produce gracias a la colaboración conjunta en interacciones directas entre las partes involucradas, partes que pueden ser los proveedores de servicio y los consumidores (Grönroos et al., 2015), o incluso otros beneficiarios (Grönroos, 2011). En la misma línea, Roser et al. (2013) aducen que la co-creación de valor es un proceso interactivo, creativo, y social iniciado por las empresas que se lleva a cabo con otros actores con el objetivo de crear valor.

Para otros autores, la co-creación es entendida como la participación y democratización de los actores inmersos en transacciones que tiene como objetivo la generación de un significado especial para ellos (Ind \& Coates, 2013), y es determinada contextualmente (Vargo \& Lusch, 2011) o como un proceso conjunto y colaborativo, que se desarrolla simultáneamente entre pares, y que tiene como resultado la producción de nuevo valor, tanto material como simbólicamente (Dalli \& Galvagno, 2013).

Con respecto a los actores que participan en la cocreación valor y la necesidad de que se desarrollen interacciones que generen co-creación de valor, encontramos diversas posiciones entre los autores. Por una parte, Vargo \& Lusch (2004) sostienen que el valor siempre es co-creado, sin embargo, Grönroos et al. (2015) y Heinonen \& Strandvik (2015) afirman que dicha tesis no es válida dado que en ocasiones, el consumidor o la empresa no están interesados en participar en la co-creación de valor, o no son conscientes del fenómeno, por lo que el valor se crea, no se co-crea. Respecto a las interacciones, Heinonen \& Strandvik (2015) sostienen que estas no son indispensables para que la co-creación de valor se produzca, lo que contradice, entre otros, a Vargo \& Lusch (2004).

Otras contradicciones entre los investigadores, fundamentales para la evolución del estudio de la cocreación de valor como constructo per-se, se encuentran entre Vargo \& Lusch (2004), Grönroos (2011) y Heinonen \& Strandvik (2015). Por una parte, Vargo \& Lusch (2004) afirman que es el servicio, en vez del producto, el aspecto que debe ser concebido como la unidad fundamental de intercambio; Grönroos (2011) a su vez expone que la base fundamental en los negocios es la recíproca creación de valor, por lo que el servicio se convierte en factor mediador, y son las interacciones, el proceso de diálogo y la influencia conjunta, los factores centrales de la co-creación. Recientemente, Heinonen \& Strandvik (2015) aseveran que la esencia de la co-creación de valor se encuentra en la lógica del consumidor, sus intenciones y acciones, así como en el valor que germina en el contexto de los consumidores.

Resumiendo, no existe todavía una definición unánime del constructo co-creación de valor, por lo que sigue vigente su discusión y controversias (Dalli \& Galvagno, 2013; Alves, Fernandes \& Raposo, 2016), siendo los aspectos más debatidos la relevancia de la intencionalidad y los comportamientos de los actores que intervienen en el proceso, así como el rol de las interacciones para que la co-creación se produzca. Pese a lo anterior queda claro en la revisión literaria, que las empresas que deseen diferenciarse de los competidores, deben reemplazar sus estrategias de marketing tradicionales centradas en el producto para concentrarse en otras fundamentadas en co-crear valor con los consumidores a través de propiciar interacciones personalizadas con su mercado (Prahalad \& Ramaswamy, 2004; Grönroos, 2008, 2011). De esta manera, a través de la co-creación de valor, se dará una respuesta adecuada a un consumidor actual caracterizado por 
estar informado, conectado a las redes, con criterio para tomar decisiones de compra en ambientes competitivos, y por su deseo de participar con la empresa en los procesos que pueda hacerlo (Prahalad \& Ramaswamy, 2004).

Por otra parte, la satisfacción es un constructo que aunque estudiado desde los años 50 e inicialmente desde la perspectiva del comportamiento del consumidor, la discusión sobre el mismo sigue también vigente (Herrera \& Gallego, 2008). La continua búsqueda por entender el constructo así como por descubrir sus antecedentes y consecuencias es de suma importancia en marketing por los demostrados efectos que tiene la satisfacción sobre la lealtad y la confianza, lo que a su vez influye en la permanencia en las relaciones (Suárez, Vázquez \& Díaz, 2007).

Una definición de gran aceptación en la academia es la de Giese \& Cote (2000), que definen el constructo como una respuesta afectiva de intensidad variable que tiene el consumidor al comprar o usar un producto, la cual tiene una duración limitada y ocurre en un momento determinado en el tiempo teniendo como referente la experiencia acumulada. Estudios posteriores afirman que, bien la satisfacción del consumidor es un estado psicológico y relativo causado por la experiencia de compra y/o consumo (Vanhamme \& Snelders, 2001), bien es un juicio psicológico que este realiza al comparar su experiencia del producto con respecto de las expectativas que tenía antes de su compra o consumo (Helgesen \& Nesset, 2007; Hunt, Geiger-Oneto \& Varca, 2012). Por su parte, Vega et al. (2013) consideran que la satisfacción del consumidor es el resultado del desempeño de quien consume, como cocreador, el servicio que la organización le presta, y la experiencia resultante de dicho comportamiento.

Si nos centramos en el ámbito universitario, la satisfacción del estudiante es señalada como un concepto paralelo al de la satisfacción y es concebida de diversas maneras en la literatura. La satisfacción es, una actitud de corto plazo producida por la evaluación subjetiva que hace el estudiante producto de sus experiencias, tanto académicas como sociales, físicas y espirituales en el campus, y de sus resultados académicos (Elliott \& Shin, 2002; Helgesen
\& Nesset, 2007); o una respuesta afectiva en un momento, la cual es el resultado de la evaluación de los servicios de enseñanza y del apoyo en el estudio que la universidad ofrece al estudiante (Palacio et al., 2002); o la percepción que tiene el estudiante con respecto de la calidad de la educación que recibe y de sus experiencias en la institución educativa (Ali \& Ahmmad, 2011).

De acuerdo con las anteriores consideraciones, la satisfacción del estudiante es trascendental porque influye en la imagen que éste tiene del programa académico que cursa y de la institución educativa, lo cual a su vez incide en su lealtad (Helgesen \& Nesset, 2007) y en la permanencia del estudiante en la universidad (Butt, 2010). Esta afirmación la reiteran para el caso de la universidad colombiana Cardona \& Bravo (2012), y para la española Schlesinger et al. $(2014,2016)$.

Estudiar la satisfacción del estudiante exige una visión sistémica que permita entender la composición interna de los elementos que en ella interactúan para dar respuesta al estudiante, quien es percibido como socio en el proceso de enseñanza-aprendizaje (Cardona \& Bravo, 2012). La satisfacción del estudiante universitario depende en gran medida de sus vivencias en la universidad (Elliott \& Shin, 2002; Helgesen \& Nesset, 2007; Ali \& Ahmmad, 2011), vivencias que se desarrollan a través de interacciones y comunicaciones de calidad con todos los recursos de la misma (Zineldin et al., 2011), lo cual denota que el consumidor, representado en este estudio por el estudiante universitario, asume comportamientos para la co-creación de valor (Prahalad \& Ramaswamy, 2004; Vargo \& Lusch, 2004; Grönroos, 2011).

Como se aprecia en la revisión teórica, la participación del consumidor en la co-creación, es de suma importancia dado que son los consumidores quienes individualmente, a través del uso de productos 0 servicios, crean valor, y su interés y compromiso en la co-creación son directamente proporcionales a su participación en actividades de co-creación (Vega et al., 2013). Adicionalmente, la participación del consumidor en la co-creación de valor ha mostrado tener influencia en la satisfacción del consumidor 
en servicios tales como turismo (Grissemann \& Stokburger, 2012) y de spa (Navarro, Llinares \& Garzon, 2016), etc., lo cual es vital para la sostenibilidad de las empresas.

Algunos estudios evidencian que los consumidores pueden asumir tres roles para la co-creación de valor: el de consultor, comunicando sus ideas, quejas y sugerencias a la empresa o a otros consumidores; el de promotor de marcas, imagen e identidad entre otras personas de sus propios círculos; y el de recurso humano, a través de su participación en el diseño y producción de nuevos productos y servicios (Bettencourt, 1997).

Yi \& Gong (2013) señalan que la co-creación puede originarse por algunos comportamientos que los autores denominan participativos y ciudadanos, pero aclarando que no existe una relación entre ellos porque siguen diferentes patrones y tienen distintos antecedentes y consecuencias (Groth, 2005). El primer comportamiento está determinado por acciones comunes y necesarias que el consumidor realiza y a través de las que se convierte en parte activa en la co-creación de valor (Groth, 2005; Yi \& Gong, 2013; Silva, Vázquez \& Revilla, 2016); actividades como cuando el consumidor acepta y sigue las sugerencias o instrucciones de la persona de la empresa con quien está en contacto o cuando interactúa personalmente con las personas del servicio (Yi \& Gong, 2013; Silva, et al., 2016). A su vez, las acciones de comportamiento ciudadano tienen un valor superior para las empresas porque son voluntarias y a través de ellas los clientes participan en la co-creación de valor para mejorar el servicio; ejemplos de ello serían: recomendar la empresa a otras personas, mostrar disposición para ayudar y asistir a otros consumidores, o tolerar incumplimientos de la firma cuando ésta no responda rápidamente a su interés o no le suministre lo que necesita, desea o espera (Yi \& Gong, 2013; Silva et al., 2016). Específicamente los comportamientos se evidencian así:

Comportamiento responsable: Los clientes que actúan responsablemente en los encuentros con las personas de las empresas se caracterizan por su disposición a seguir las recomendaciones y sugerencias del personal del servicio y actuar cooperativamente en el proceso de co-creación de valor cumpliendo sus deberes (Bettencourt, 1997; Elsharnouby, 2015; Navarro et al., 2016; Silva et al., 2016). En el ambiente universitario entendemos que este comportamiento es igualmente necesario para que el estudiante logre su objetivo de formación sin contratiempos, y se lleva a cabo cuando el estudiante acata instrucciones impartidas por docentes y otros empleados de la universidad, cuando cumple con sus tareas y cuando participa colaborativamente en las diversas actividades del proceso de enseñanza-aprendizaje, contribuyendo así a que la educación sea co-creada.

Interacción personal: Estos comportamientos hacen referencia a la cortesía, amabilidad y respeto que debe tener el consumidor, en nuestro caso el estudiante universitario, al relacionarse con las personas encargadas de ofrecer el servicio (empleados de la universidad), lo cual constituye una base primordial para la co-creación de valor (Elsharnouby, 2015; Silva et al., 2016).

Recomendar: Esta es una acción voluntaria del consumidor que se refleja en acciones tales como hablar bien de una empresa que le presta un servicio, sugerir el uso de sus servicios entre conocidos y familiares, y contribuir a la imagen de la empresa a través de la propagación de comentarios positivos (Elsharnouby, 2015; Navarro et al., 2016; Silva et al., 2016), lo que se observa por ejemplo en el contexto de la universidad, cuando el estudiante habla bien de su universidad e invita a otras personas a escoger dicha universidad ante otras opciones.

Disposición para ayudar: Esta acción voluntaria se presenta, por ejemplo, cuando un consumidor ayuda a otros consumidores en sus elecciones $\mathrm{O}$ necesidades sin la intervención de empleados (Elsharnouby, 2015; Silva et al., 2016), cuando los estudiantes explican a sus compañeros los temas que no entienden o los ayudan en un ejercicio, o los aconsejan cuando lo necesitan.

Tolerancia: Los consumidores son tolerantes cuando aceptan con paciencia fallos en el servicio que el proveedor ofrece, o cuando los empleados cometen errores, o en las ocasiones en que deben esperar más tiempo del previsto (Elsharnouby, 2015; Navarro et al., 2016; Silva et al., 2016). El estudiante 
universitario se ve enfrentado a situaciones imprevistas con relativa frecuencia, tales como cancelación de clases, y al mostrar paciencia y ser flexible ante los cambios asume este comportamiento que es muy importante para la co-creación de valor.

En consecuencia, teniendo como referencia la teoría analizada y siguiendo a Vega et al. (2013) y Navarro et al. (2016), que han estudiado la influencia de las actuaciones de co-creación de valor del consumidor sugeridas por Yi \& Gong (2013) en la satisfacción en los contextos de servicios, proponemos en esta investigación, que los comportamientos de co-creación de valor que el estudiante universitario asume en su vida universitaria influyen en la satisfacción que percibe con el servicio prestado por la institución de educación superior donde cursa sus estudios, lo que nos lleva a plantear las siguientes hipótesis:

H1. Existe relación directa y positiva entre el comportamiento responsable del estudiante universitario en su universidad y la satisfacción del estudiante con la universidad.

H2. Existe relación directa y positiva entre el comportamiento de interacción personal del estudiante universitario en su universidad y la satisfacción del estudiante con la universidad.

H3. Existe relación directa y positiva entre las recomendaciones que el estudiante universitario hace de la universidad donde estudia y su satisfacción con la universidad.

H4. Existe relación directa y positiva entre el comportamiento del estudiante universitario de ayuda a otros estudiantes de la universidad y la satisfacción del estudiante con la universidad.

H5. Existe relación directa y positiva entre la tolerancia que muestre el estudiante universitario en la universidad donde estudia y la satisfacción que el estudiante percibe con su universidad.

\section{Metodología}

El objetivo general de nuestra investigación fue determinar si existe una relación positiva y significati- va entre actuaciones de estudiantes universitarios, como elementos sustanciales de la co-creación de valor, y la satisfacción de dichos estudiantes con el servicio percibido de la universidad donde cursan sus estudios. Dado que los constructos de los comportamientos del consumidor que determinan la co-creación de valor siguen diferentes patrones $y$ tienen distintos antecedentes y consecuencias (Groth, 2005), en esta investigación se analizó la influencia de cada uno de los cinco componentes de co-creación de valor de la escala de satisfacción percibida por los estudiantes universitarios, lo que está en línea con el trabajo realizado por Navarro et al., (2016) en el contexto de servicios de belleza en España. La Ilustración 1 muestra el modelo de relaciones propuesto.

llustración 1. Modelo de relaciones estructurales propuestas

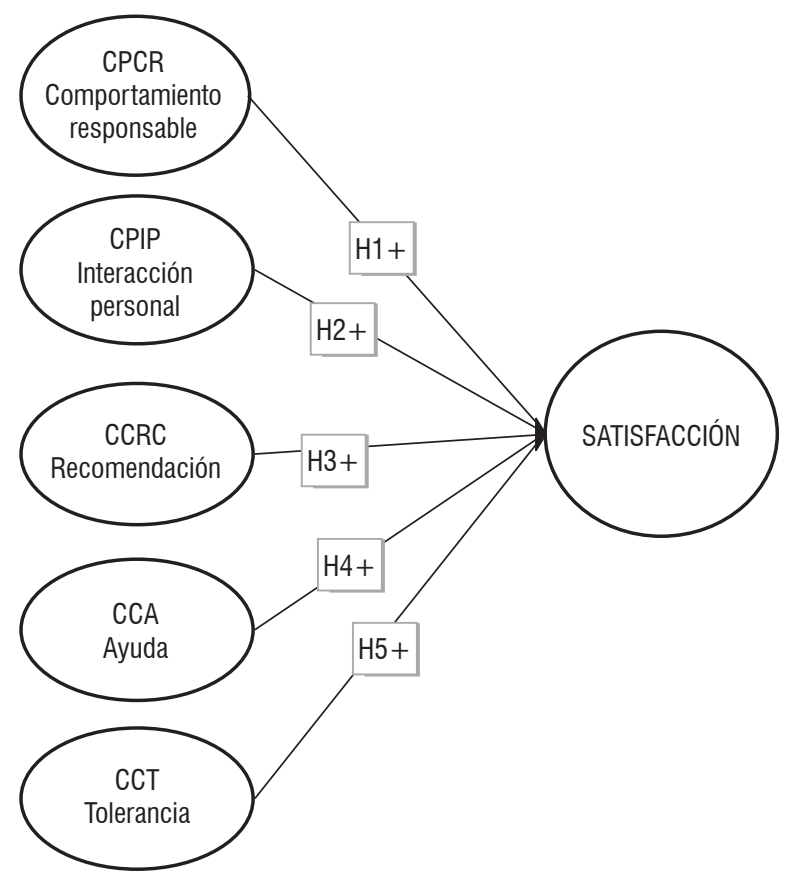

Fuente: Adaptación de Navarro et al. (2016).

La muestra estuvo conformada por 320 estudiantes colombianos de pregrado (51\% hombres y el $49 \%$ mujeres) matriculados en la Universidad de Ibagué, que estaban participando en diversos cursos interdisciplinarios de la Universidad en el marco de la Escue- 
la Internacional de Verano en el 2014. Las edades del $44 \%$ de los estudiantes oscilaban entre los 17 y los 20 años, el $38 \%$ entre los 21 y los 23 , y el $18 \%$ era mayor de 23 años. Los jóvenes estaban además matriculados en carreras profesionales de distintas facultades de dicha universidad: Humanidades, Ingeniería y Ciencias Económicas y Administrativas.

Los cuestionarios fueron aplicados por una de las investigadoras en las aulas de clase, y antes de empezar su diligenciamiento se explicó el instrumento en detalle para asegurar la correcta interpretación de las preguntas. Las pocas dudas que los estudiantes tuvieran fueron aclaradas durante la jornada de recolección de la información. La ficha técnica del estudio se recoge en la Tabla 1 . La información fue recolectada entre el 24 de junio y el 4 de julio del año 2014 a través de encuestas personales, para lo cual se diseñó un cuestionario estructurado compuesto por 35 preguntas cerradas, 29 de las cuales miden la participación del consumidor en la co-creación de valor (Yi \& Gong, 2013) y seis estaban relacionadas con la satisfacción (Suárez et al., 2007).

Tabla 1. Ficha técnica del estudio

\begin{tabular}{|c|c|}
\hline Universo & $\begin{array}{l}\text { Número de estudiantes de pregrado de la } \\
\text { Universidad de Ibagué- Colombia }\end{array}$ \\
\hline Ámbito & Ibagué - Colombia \\
\hline Unidad de Análisis & $\begin{array}{l}\text { Estudiantes de cursos de verano de todas } \\
\text { las facultades de la Universidad }\end{array}$ \\
\hline $\begin{array}{l}\text { Selección de elementos del } \\
\text { Universo }\end{array}$ & $\begin{array}{l}\text { Listado de los estudiantes que participan } \\
\text { en los cursos de verano } \\
\text { (Humanidades, Económicas, Ingenierías) }\end{array}$ \\
\hline Muestra obtenida & 320 \\
\hline Procedimiento muestral & Muestreo aleatorio simple \\
\hline Nivel de error & $p=q=0,5 ; 5 \%$ \\
\hline Recogida de la Información & $\begin{array}{l}\text { Encuesta estructurada aplicada por una } \\
\text { de las investigadoras en los salones de } \\
\text { clases }\end{array}$ \\
\hline Fechas del trabajo de campo & Entre el 24 de junio y el 4 de julio de 2014 \\
\hline
\end{tabular}

Fuente: Elaboración propia.

En esta investigación se pretendió detectar si los comportamientos del estudiante universitario que contribuyen a la co-creación de valor influyen en la satisfacción del estudiante con el servicio que la uni- versidad le presta, para lo cual se utilizaron las escalas de medición sugeridas por Silva et al. (2016) y la de Suárez et al., (2007), las cuales miden respectivamente, co-creación de valor desde la perspectiva del consumidor, y satisfacción (Ilustración 1).

Vega et al. (2013), así como Navarro et al. (2016) han estudiado la relación entre el comportamiento del consumidor en la co-creación de valor y la satisfacción en servicios de belleza y de spa, respectivamente, usando las escalas de co-creación de Yi \& Gong (2013) y de satisfacción de Suárez et al. (2007). La escala de co-creación de Silva et al. (2016) es una adaptación testada de la escala de Yi \& Gong (2013) por lo que la encontramos muy adecuada para los fines de este estudio. Esta escala está conformada por 15 ítems agrupados en cinco factores que miden comportamientos esperados y voluntarios que influyen en la co-creación de valor: comportamiento responsable, interacción personal, recomendación, ayuda, y tolerancia.

Por su parte, la satisfacción fue medida con la escala de seis ítems propuesta por Suárez et al. (2007), que ha sido ampliamente validada y utilizada para medir el constructo satisfacción en el sector de servicios; por ejemplo, en turismo por Suárez et al. (2007), en salones de belleza y cuidado personal por Vega et al. (2013), y más recientemente en servicio de spa por Navarro et al. (2016). Esta escala tiene como objetivo evaluar la satisfacción global del consumidor con la empresa e incluye y hace referencia a la satisfacción pasada. En tal sentido, se entiende la satisfacción como una variable acumulativa que comprende la satisfacción de los consumidores a través de una variedad de encuentros de servicio (Vega et al. 2013), algo que sucede en la relación entre el estudiante universitario y la institución de educación superior donde éste cursa sus estudios. Las mediciones se realizaron con una escala de Likert de cinco puntos que van desde totalmente en desacuerdo hasta completamente de acuerdo.

\section{Análisis de datos y resultados}

Para el procesamiento y análisis de la información recolectada se utilizaron los programas SPSS ver- 
sión 15.00 y EQS 6.2 para Windows, lo que hizo posible determinar la validez y fiabilidad del instrumento a través de Análisis Factorial Confirmatorio (AFC), así como comprobar las hipótesis a través del Sistema de Ecuaciones Estructurales (SEM).

Para empezar, se realizó un Análisis Factorial Exploratorio en SPSS versión 15.00 a la escala que mide la co-creación de valor desde la perspectiva del consumidor creada por Yi \& Gong (2013), encontrando que la escala es válida y fiable. Los resultados de la medida de adecuación muestral de Kaiser-MeyerOlkin y la prueba de esfericidad de Barlett fueron 0,863 y 0,00 respectivamente, de lo que se deduce que la muestra de datos es suficiente para soportar un análisis confirmatorio. Seguidamente se revisó la Varianza Total Explicada para determinar el número de factores al que se puede reducir el instrumento. Dado que la varianza acumulada es del 53.65\%, se asume que las ocho variables latentes se pueden mantener. Al analizar la Matriz de Factores Rotados se aprecia que se mantiene la configuración de las variables observadas con 7 de los factores y que del factor 1 solo una de las variables es representativa, por lo que decidimos eliminar las dos variables no representativas. Al analizar el AFE sin las dos preguntas observamos que la varianza acumulada mejoró de 53,65\% a 56,40\%, lo que denota la validez del instrumento de medida.

La validez de contenido se aseguró a través de la revisión teórica de los constructos co-creación, satisfacción y de la realizada por los autores que proponen la escala de co-creación.

Con los datos obtenidos del AFE procedimos a analizar la validez y confiabilidad de instrumento de medida a través de un Análisis Factorial Confirmatorio AFC. La información de las medidas de bondad de ajuste, así como de la fiabilidad y la validez convergente de la escala de co-creación se aprecia en la tabla 2. Las medidas de la bondad de ajuste arrojaron buenos resultados. El cociente entre los grados de libertad $(174)$ y el Chi cuadrado $(313,89)$ están dentro de los parámetros sugeridos por Hair et al. (1998) e indican que los datos son favorables y están centrados. Adicionalmente, tanto la media de todos los residuos $(0,0336)$, como la media sin tener en cuenta la diagonal $(0,0370)$, son muy inferiores a 0,08 , lo cual indica que la diferencia entre la realidad y la matriz estimada es mínima. En el mismo sentido, el índice de ajuste no normalizado (NNFI) de 0,95 es bueno al ser superior al 0,90 propuesto (Schumacker \& Lomax, 1996), como lo es también el índice comparativo de ajuste (CFI) de 0,96 al ser superior a 0,95 sugerido (Hu \& Bentler, 1999), y el indicador de parsimonia (RMSEA) de 0,05 denota un ajuste aceptable (Browne \& Cudeck, 1993).

Seguidamente, para probar la validez convergente del instrumento, se comprobó que todas las cargas de los ítems de las variables fueran significativas y que los tamaños de las mismas se acercaran o sobrepasaran el valor de 0,70 sugerido por Hair et al. (1998). En lo que respecta a la fiabilidad compuesta (CR) y la varianza extraída (AVE), todos los resultados fueron positivos, ya que todos los indicadores de CR fueron muy superiores a lo propuesto de 0,70 (Fornell \& Larcker, 1981) y los de AVE estuvieron por encima del 0,50 establecido (Fornell \& Larcker, 1981). En consecuencia, los resultados demuestran la fiabilidad del instrumento de medida.

Al igual que lo realizado por Silva et al. (2016) y por Elsharnouby $(2015,2016)$ que utilizaron la escala de co-creación desde la óptica del comportamiento del consumidor, la validez discriminante del instrumento de medida se demostró al comprobar que el cuadrado de la correlación entre cada par de factores es inferior que el AVE de cada factor, lo cual cumple con lo sugerido por Fornell \& Larcker (1981) tal como se aprecia en la tabla 3.

Adicionalmente, al calcular la varianza total explicada de la escala de la satisfacción, se constató que solamente uno de los seis ítems presenta valor superior a 1 y explica el $53 \%$ de la varianza, lo que indica que la validez de la escala es unidimensional. De manera similar se detectó en la Matriz Factorial que todas las cargas estandarizadas son superiores a 0,627 . Estos resultados permiten inferir la validez de la escala escogida para medir la satisfacción. 
Tabla 2. Validación del instrumento de medida-Fiabilidad y validez convergente

\begin{tabular}{|c|c|c|c|c|c|}
\hline Factores & Ítems & Cargas estandarizadas & $\mathbf{T}$ & CR & AVE \\
\hline \multirow{4}{*}{ F1 Comportamiento responsable } & CPCR8 & $0,73^{\star *}$ & 13,97 & 0,82 & 0,54 \\
\hline & CPCR9 & $0,75^{\star \star}$ & 14,54 & & \\
\hline & CPCR10 & $0,77^{\star \star}$ & 15,23 & & \\
\hline & CPCR11 & $0,68^{* \star}$ & 12,85 & & \\
\hline \multirow{3}{*}{ F2 Interacción personal } & CPIP12 & $0,82^{\star \star}$ & 17,45 & 0,90 & 0,75 \\
\hline & CPIP13 & $0,94^{\star \star}$ & 21,22 & & \\
\hline & CPIP15 & $0,83^{* *}$ & 17,20 & & \\
\hline \multirow{3}{*}{ F3 Recomendación } & CCRC20 & $0,86^{* \star}$ & 18,74 & 0,90 & 0,75 \\
\hline & CCRC21 & $0,93^{* *}$ & 21,01 & & \\
\hline & CCRC22 & 0,80 ** & 16,68 & & \\
\hline \multirow{2}{*}{ F4 Ayuda } & CCA23 & $0,84^{\star *}$ & 15,57 & 0,84 & 0,72 \\
\hline & CCA24 & $0,86^{* *}$ & 16,04 & & \\
\hline \multirow{3}{*}{ F5 Tolerancia } & ССТ27 & $0,63^{\star *}$ & 11,06 & 0,77 & 0,53 \\
\hline & ССТ28 & $0,74^{* *}$ & 13,25 & & \\
\hline & ССТ29 & $0,81^{\star \star}$ & 14,51 & & \\
\hline
\end{tabular}

Fuente: Elaboración propia.

Tabla 3. Validez discriminante instrumento de medida

\begin{tabular}{|c|c|c|c|c|c|c|}
\hline & F1 CPCR & F2 CPIP & F3 CCRC & F4 CCA & F5 CCT & F6 SAT \\
\hline F1 CPCR & 0,54 & & & & & \\
\hline F2 CPIP & 0,30 & 0,75 & & & & \\
\hline F3 CCRC & 0,27 & 0,21 & 0,75 & & & \\
\hline F4 CCA & 0,35 & 0,20 & 0,17 & 0,72 & & \\
\hline F5 CCT & 0,00 & 0,06 & 0,10 & 0,02 & 0,53 & 0,15 \\
\hline F6 SAT & 0,13 & 0,10 & 0,41 & 0,13 & & 0,53 \\
\hline
\end{tabular}

Fuente: Elaboración propia.

Tras haber constatado las bondades psicométricas del instrumento de medida, se estimó el modelo estructural de la Ilustración 1 para comprobar las relaciones allí sugeridas. En la parte inferior de la tabla
4 se aprecia que los valores de los indicadores de la bondad de ajuste del modelo arrojados por el modelo de ecuaciones estructurales SEM son superiores a los recomendados, lo que los hace muy aceptables. 
En la tabla 4 y en la Ilustración 2 se evidencia que aunque cuatro de los parámetros arrojaron resultados positivos, dos de ellos no son significativos. En consecuencia, las hipótesis tres y cinco son aceptadas, es decir, los comportamientos del estudiante de recomendar la universidad y de ser tolerante con las inconsistencias que pueda encontrar en su universidad, influyen directa y positivamente en su sa- tisfacción con el servicio que recibe de la institución educativa. Contrariamente, la primera, segunda y cuarta hipótesis son rechazadas, lo que indica que ni el comportamiento responsable, ni la interacción personal, ni las actuaciones del estudiante que denotan disposición de ayudar, influyen en la satisfacción que el estudiante percibe con el servicio de su universidad.

Tabla 4. Comprobación de hipótesis

\begin{tabular}{|c|c|c|c|c|c|}
\hline Hipótesis & Relación estructural planteada & & $\beta$ estandarizada & $t$ & Resultado \\
\hline H1. & Comportamiento responsable (CPCR) & $\longrightarrow$ Satisfacción & 0,044 & 0,52 & Rechazada \\
\hline $\mathrm{H} 2$. & Interacción personal (CPIP) & $\longrightarrow$ Satisfacción & -0.044 & $-0,66$ & Rechazada \\
\hline H3. & Recomendación (CCRC) & $\longrightarrow$ Satisfacción & $0,534^{* *}$ & 6,74 & Aceptada \\
\hline $\mathrm{H} 4$. & Ayuda (CCA) & $\longrightarrow$ Satisfacción & 0,092 & 1,28 & Rechazada \\
\hline H5. & Tolerancia (CCT) & $\longrightarrow$ Satisfacción & $0,217^{*}$ & 3,37 & Aceptada \\
\hline
\end{tabular}

Fuente: Elaboración propia.

Ilustración 2. Modelo contrastado

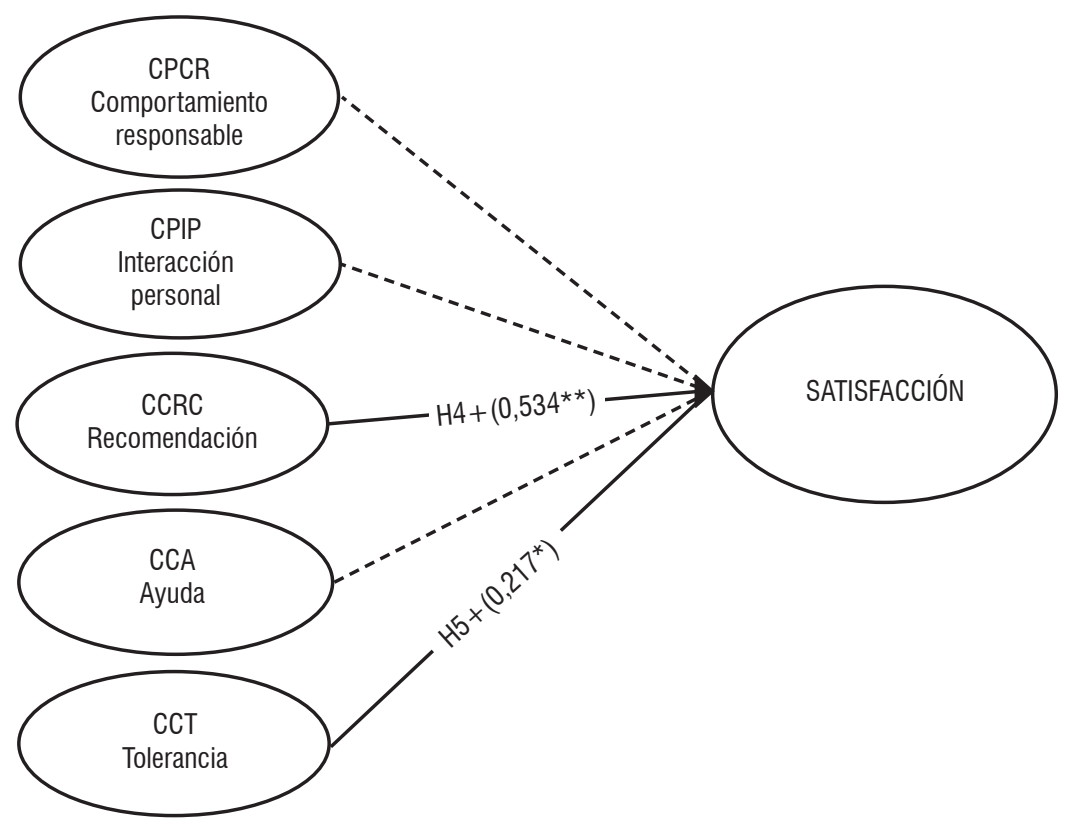

Fuente: Elaboración propia. 


\section{Conclusiones}

El objetivo de este trabajo de investigación fue determinar si la co-creación de valor desde la perspectiva del consumidor, influye en la satisfacción del estudiante con los servicios prestados por su universidad. Entendemos que el consumidor, en este caso específico, está representado por el estudiante universitario; en consecuencia, las hipótesis formuladas buscaban identificar si los comportamientos que el estudiante universitario asume para la co-creación de valor en el entorno de enseñanza, inciden en la satisfacción que éste percibe con la universidad donde cursa sus estudios. Para lograr el objetivo se aplicó personalmente una encuesta a estudiantes en sus aulas de clase en una universidad colombiana.

El estudio de la co-creación de valor como antecedente de la satisfacción ha sido un fenómeno escasamente explorado en la literatura del marketing, a la fecha, que tengamos conocimiento, tan solo dos estudios han sido publicados en los que se analiza esta relación en el mismo sentido aunque para otro tipo de servicios por Vega et al. (2013) y Navarro et al. (2016), ambos en España. En consecuencia, las contribuciones académicas de esta investigación son tres y radican en, que por primera vez se estudia la relación entre la co-creación de valor desde la perspectiva del consumidor y la satisfacción en el contexto universitario, que el estudio se realiza en un país latinoamericano, y que además se testa una escala adaptada al contexto español recientemente publicada.

Los resultados del estudio determinaron que dos de las cinco hipótesis se cumplieron, lo que permite establecer algunas conclusiones y proponer implicaciones a los responsables de la gestión en instituciones universitarias, lo que es valioso si se tiene en cuenta que este servicio es ofrecido por un alto número de instituciones interesadas en permanecer y mejorar su participación, en el mercado de los servicios de la educación superior.

Los hallazgos indican que los comportamientos de co-creación de valor del estudiante universitario al recomendar la universidad a otras personas y ser tolerantes ante imprevistos que afectan la calidad del servicio esperado de la universidad, influyen directa y positivamente en su satisfacción. Es interesante notar que estos dos comportamientos son voluntarios, lo que los hace más valiosos para la universidad.

Con respecto al primer comportamiento, cuando el estudiante recomienda los servicios de su universidad $e$ invita a amigos y familiares a ser parte de la misma y a utilizar sus servicios, se convierte en promotor voluntario de su universidad (Elsharnouby, 2015). Este aspecto es muy importante, si se tiene en cuenta que los estudiantes expresan sus opiniones de manera personal y a través de medios virtuales, lo que les permite que su mensaje se propague rápidamente entre sus contactos. Por otro lado, el poder de convicción que tiene un estudiante entre sus contemporáneos es más alto que el del personal propio de las universidades. En consecuencia, las universidades deben diseñar e implementar estrategias que les facilite detectar y acercarse a estos estudiantes, y convertirlos en promotores de los servicios de sus universidades para atraer nuevos estudiantes, mantener contacto con los egresados, y posicionar sus claustros.

El segundo comportamiento de co-creación del estudiante universitario que mostró influir en su satisfacción con la universidad es la tolerancia, lo que es interesante si se tiene en cuenta que en el servicio universitario se presentan, con frecuencia, imprevistos -trabajos extraordinarios, cancelación de clases, mal funcionamiento de las tecnologías, etc.- que pueden afectar la buena calidad del servicio. Los estudiantes que reaccionan con paciencia en dichas ocasiones se convierten en aliados de la universidad $y$ pueden influir en las reacciones de sus compañeros de clase. En consecuencia, es muy importante que los funcionarios de la universidad identifiquen a estos estudiantes y establezcan estrategias para hacerlos participativos en el proceso de prestación del servicio, por ejemplo nombrándolos monitores o asignándoles algunas labores que les permita sentirse valiosos y contribuir a la mejora del buen ambiente en su universidad.

Es preciso resaltar, sin embargo, que ni el comportamiento responsable, ni la interacción personal, ni 
la intención de ayudar resultaron influir en la satisfacción del estudiante. Este descubrimiento nos lleva a deducir que los estudiantes no son conscientes de que acciones -como cumplir con sus labores en la universidad, atender las instrucciones de los profesores y otro personal del servicio de la universidad, guardar relaciones respetuosas, y ayudar a sus compañeros cuando se necesita-, hacen que su grado de satisfacción aumente al obtener mejores resultados académicos y al hacer de la universidad un espacio en el cual pueden encontrar apoyo y experimentar solidaridad.

Como reflexión, consideramos que los directivos de universidades deben ser conscientes de que las instituciones de educación superior contemporáneas están llamadas a competir entre sí no solamente con educación de calidad, sino con buen servicio, propiciando interacciones a través de programas permanentes que permitan a sus estudiantes sentirse importantes, escuchados y valorados como cocreadores del servicio de educación, o como socios en el proceso de enseñanza-aprendizaje (Cardona \& Bravo, 2012). De esta manera, es indispensable crear un ambiente universitario que posibilite interacciones positivas estudiante-universidad, dado que está demostrado que la calidad de dichas interacciones influye en la imagen que los estudiantes perciben de su universidad y en que sientan satisfacción con el claustro (Díaz et al., 2007); satisfacción que a la vez influye en la lealtad del estudiante (Helgesen \& Nesset, 2007) y en su permanencia en la institución (Butt, 2010; Cardona \& Bravo, 2012; Díaz et al., 2007).

Concluyendo, las universidades deben asumir dos grandes retos. En primer lugar, cambiar el paradigma de concebir la universidad como una organización proveedora de conocimientos, en la que los estudiantes participan como receptores de enseñanzas, por una concepción de orientación al servicio, en la que se reconozca a la universidad como un centro educativo cuyo principal propósito es generar interacciones de calidad entre estudiantes y empleados de todas las áreas de la universidad, teniendo en cuenta que la satisfacción que los estudiantes perciben con la institución es resultante de sus vivencias globales en el claustro (Elliott \& Shin,
2002; Palacio et al., 2002; Ali \& Ahmmad, 2011). $\mathrm{Y}$ en segundo lugar, en un ambiente competitivo y globalizado como el actual, las universidades se ven abocadas a invertir recursos de diversa índole, para generar ambientes variados que satisfagan a sus estudiantes y les estimulen a recomendar la universidad, a permanecer en el claustro y a mantener sus lazos a largo plazo.

Este estudio presenta algunas limitaciones. Primero, como el estudio del constructo co-creación de valor desde la perspectiva del consumidor es tan reciente, a la fecha ha sido creada tan solo una escala para realizar su medición, la escala de Yi \& Gong (2013), escala que fue recientemente adaptada para el contexto español por Silva et al. (2016). Decidimos utilizar esta última porque al haber realizado previamente múltiples análisis estadísticos con la de Yi \& Gong (2013) en nuestra investigación, tuvimos serios inconvenientes, en las mediciones de fiablidad del instrumento en los mismos factores, y en la mayoría de los ítems que Silva et al. (2016) decidieron eliminar después de su riguroso análisis. Como resultado, encontramos que la escala de Silva et al. (2016) arrojó excelentes resultados de fiabilidad y validez al ser aplicada en el contexto de la educación superior en Colombia. En consecuencia, proponemos que futuras investigaciones se orienten a testar y a mejorar la escala para medir la co-creación de valor desde la perspectiva del consumidor en diversos entornos de servicios y culturales, ámbito de estudio que se encuentra en su incipiente comienzo.

Para terminar, la revisión de literatura realizada en esta investigación nos permite deducir que son necesarios más estudios que permitan descubrir los antecedentes y las consecuencias del comportamiento del consumidor para la co-creación de valor en el ámbito de la educación superior, por lo que sugerimos que estas relaciones sean exploradas en futuras investigaciones.

\section{Referencias}

Ali, A. \& Ahmmad, I. (2011). Key Factors for Determining Students' Satisfaction in Distance Learning Courses: A Study of Allama Iqbal Open University. Contemporary Educational Tecchnology, 2(2), 118-134. 
Alves, H., Fernandes, C. \& Raposo, M. (2016). Value co-creation: Concept and contexts of application and study. Journal of Business Research, 69(5), 1626-1633.

Ballantyne, D. \& Varey, R. (2008). The service-dominant logic and the future of marketing. Journal of the Academy of Marketing Science, 36(1), 11-14.

Bettencourt, L. (1997). Customer voluntary performance: Customers as partners in service delivery. Journal of Retailing, 73(3), 383-406.

Bettencourt, L., Lusch, R. \& Vargo, S. (2014). A Service Lens on Value Creation. California management review, 57(1), 44-66.

Browne, M. \& Cudeck, R. (1993). Alternative ways of assessing model fit. California, Estados Unidos: Sague Focus Editions.

Butt, B. (2010). A study examining the students satisfaction in higher education. Procedia-Social and Behavioral Sciences, 2(2), 5446-5450.

Cardona, M. \& Bravo, J. (2012). Service quality perceptions in higher education institutions: the case of a colombian university. Estudios Gerenciales, 28(125), 23-29.

Dalli, D. \& Galvagno, M. (2013). The literature on co-creation: bibliometric analysis and review. In AMA Summer Marketing Educators' Conference Proceedings.

Díaz, A., Suárez, L. \& Vásquez, R. (2007). La confianza y la satisfacción del cliente. Revista europea de dirección y economía de la empresa, 16(3), 115-132.

Elsharnouby, T. (2015). Student co-creation behavior in higher education: the role of satisfaction with the university experience. Journal of Marketing for Higher Education, 25(2), 238-262.

Elsharnouby, T. (2016). Participation behaviour among international students: the role of satisfaction with service augmentation and brand choice attainment. International Journal of Educational Management, 30(5).

Elliott, K. \& Shin, D. (2002). Student satisfaction: an alternative approach to assessing this important concept. Journal of Higher Education Policy and Management, 24(2), 197-209.

Fornell, C. \& Larcker, D. (1981). Structural equation models with unobservable variables and measurement error: Algebra and statistics. Journal of Marketing research, 18(3), 382-388.

Giese, J. \& Cote, J. (2000). Defining Consumer Satisfaction. Academy Of marketing science review, 1(1), 1-22.

González, M., Pino, M. \& Penado, M. (2016). Estudio de la satisfacción percibida por los estudiantes de la UNED con su vida universitaria. RIED. Revista Iberoamericana de Educación a Distancia, 20(1).

Grissemann, U. \& Stokburger, N. (2012). Customer co-creation of travel services: The role of company support and customer satisfaction with the co-creation performance. Tourism Management, 33(6), 1483-1492.

Grönroos, C. (2008). Service logic revisited: ¿who creates value?, and ¿who co-creates? European Business Review, 20(4), 298-314.

Grönroos, C. (2011). Value co-creation in service logic: A critical analysis. Marketing Theory, 11(3), 279-301.
Grönroos, C., Strandvik, T. \& Heinonen, K. (2015). Value CoCreation: Critical Reflections. The Nordic School, 69.

Grönroos, C. \& Voima, P. (2013). Critical service logic: making sense of value creation and co-creation. Journal of the Academy of Marketing Science, 41(2), 133-150.

Groth, M. (2005). Customers as Good Soldiers:Examining Citizenship Behaviors. Journal of Management, (31), 7-27.

Gummesson, E. \& Mele, C. (2010). Marketing as value co-creation through network interaction and resource integration. Journal of Business Market Management, 4(4), 181-198.

Hair, J., Anderson, R., Tatham, R., Black, W. \& Babin, B. (1998). Multivariate data analysis with readings. Sydney, Australia: Prentice Hall.

Heinonen, K. \& Strandvik, T. (2015). Customer-dominant logic: foundations and implications. Journal of Services Marketing, 29(6/7), 472-484.

Helgesen, O. \& Nesset, E. (2007). Images, satisfaction and antecedents: ¿Drivers of student loyalty? A Case study of a Norwegian University College. Corporate Reputation Review, 10(1), 38-59.

Herrera, A. \& Gallego, S. (2008). Efectos de la satisfacción emocional y evaluativa de los espectadores deportivos sobre sus intenciones de asistir y recomendar. Innovar: Revista de ciencias administrativas y sociales, 18(32), 75-86.

Hu, L. \& Bentler, P. (1999). Cutoff criteria for fit indexes in covariance structure analysis: Conventional criteria versus new alternatives. A Multidisciplinary Journal, 6(1), 1-55.

Hunt, D., Geiger-Oneto, S. \& Varca, P. (2012). Satisfaction in the context of customer co production: a behavioral involvement perspective. Journal of Consumer Behaviour, 11(5), 347-356.

Ind, N. \& Coates, N. (2013). The meanings of co-creation. European Business Review, 25(1), 86-95.

Jurkowitsch, S., Vignali, C. \& Kaufmann, H-R. (2006). A student satisfaction model for Austrian higher education providers considering aspects of marketing communications. Innovative Marketing, 3 (Special Edition).

Navarro, S., Llinares, C. \& Garzon, D. (2016). Exploring the relationship between co-creation and satisfaction using QCA. Journal of Business Research, 69(4), 1336-1339.

Neghina, C., Caniëls, M., Bloemer, J. \& van Birgelen, M. (2015). Value cocreation in service interactions Dimensions and antecedents. Marketing Theory, 15(2), 221-242.

Palacio, A., Meneses, G. \& Pérez, P. (2002). The configuration of the university image and its relationship with the satisfaction of students. Journal of Educational Administration, 40(5), 486-504.

Payne, A., Storbacka, K. \& Frow, P. (2008). Managing the cocreation of value. Academy of Marketing Science, 36(1), 83-96.

Prahalad, C. \& Ramaswamy, C. (2004). Co-creation experiences: The next practice in value creation. Journal of interative marketing, 18(3), 5-14.

Roser, T., DeFillippi, R. \& Samson, A. (2013). Managing your co-creation mix: co-creation ventures in distinctive contexts. European business review, 25(1), 20-41. 


\section{COMPORTAMIENTO DEL CONSUMIDOR EN LA CO-CREACIÓN DE VALOR Y SU RELACIÓN CON LA SATISFACCIÓN \\ EN EL ENTORNO UNIVERSITARIO: UNA APLICACIÓN A LA UNIVERSIDAD DE IBAGUÉ (COLOMBIA)}

Saarijärvi, H., Kannan, P. \& Kuusela, H. (2013). Value co-creation: theoretical approaches and practical implications. European Business Review, 25(1), 6-19.

Schlesinger, M., Cervera Taulet, A., Iniesta Bonillo, M. \& Sánchez Fernández, R. (2014). Un enfoque de marketing de relaciones a la educación como un servicio: aplicación a la Universidad de Valencia. Revista de Ciencias administrativas y Sociales Innovar, 24(53), 113-125.

Schlesinger, W., Cervera, A. \& Pérez, C. (2016) Sticking with your university: the importance of satisfaction, trust, image, and shared values, Studies in Higher Education, 1-17.

Schumacker, R. \& Lomax, R. (1996). A beginner's guide to structural equation modeling. New Jersey, New Jersey, USA: Paperback.

Silva, F., Vázquez, M. \& Revilla, M. (2016). La percepción del cliente sobre la co-creación de valor. Adecuación de la escala de Yi y Gong al contexto español. Esic market, (153), 9-40.

Suárez, L., Vázquez, R. \& Díaz, A. (2007). La confianza y la satisfacción del cliente: variables clave en el sector turístico.
Revista europea de dirección y economía de la empresa, 16(3), 115-132.

Vanhamme, J. \& Snelders, D. (2001). The role of surprise in satisfaction judgments. Journal of consumer satisfaction dissatisfation and complaning behavior, (14), 27-45.

Vargo, S. \& Lusch, R. (2004). Envolving to a new dominant logic for marketing. Journal of marketing, 1-17.

Vargo, S. \& Lusch, R. (2011). It's all B2B ... and beyond: Toward a systems perspective of the market. Industrial Marketing Management, 40(2), 181-187.

Vega, M., Revilla, M. \& Cossío, F. (2013). The value co-creation process as a determinant of customer satisfaction. Management Decision, 51(19), 1945-1953.

Yi, Y. \& Gong, T. (2013). Customer value co-creation behavior: scale development and validation. Journal of Business Research, 66(9), 1279-1284.

Zineldin, M., Akdag, H. \& Vasicheva, V. (2011). Assessing quality in higher education: new criteria for evaluating students' satisfaction. Quality in Higher Education, 17(2), 231-243. 